$\mathrm{J}$ o u r n a $\mathrm{l}$ of

Mathematics

and Applications

JMA No 39, pp 47-58 (2016)

\title{
On the Evolution of Academic Staff Structure in a University Setting
}

\author{
Virtue U. Ekhosuehi
}

\begin{abstract}
This paper models the academic staff structure in a university as a system of stocks and flows in a three-dimensional space, $\mathbb{R}^{3}$. The stocks are the number of academic staff in a particular state at a given time and the flows are the staff moving between any two states over an interval of time. The paper places emphasis on the grade-specific completion rates of Graduate Assistants, who choose to study in the university in which they are employed for higher degrees. The study describes the evolution of structures in the university as a linear recurrence system. Some aspects of linear algebra are employed as a theoretical underpinning to gain insights into the transformation matrix of the recurrence system. A number of resulting propositions are presented along with their proofs. We provide two theorems to serve as a means of predicting a university manpower structure. Following that a numerical illustration of the theorems and propositions is provided with data which are representative of the kind of data in a Nigerian university system.
\end{abstract}

AMS Subject Classification: 15A18, 91D35.

Keywords and Phrases: Diagonalisable matrices; Eigenvalues; Linear mapping; Manpower planning; Recurrence system.

\section{Introduction}

The evolution of staff structure in a manpower system is a key issue attracting the attention of management, planners and policy makers, particularly in a university system. This is because the future distribution of staff could aid management in planning and resource allocation, which may include framing policies to guide against shortages and excess staffing requirements and budgeting for personnel costs and staff development programmes. The population of academic staff in a university is viewed as a system of interconnected states in such fashion that each individual academic staff can follow a path to become a professor. In this light a university manpower

COPYRIGHT (c) by Publishing House of Rzeszów University of Technology P.O. Box 85, 35-959 Rzeszów, Poland 
system may be called a hierarchical system. Authors such as Vassiliou and Tsantas [17], Guerry [6] and Kipouridis and Tsaklidis [8] analyzed hierarchical manpower systems using discrete-time Markov chains. The semi-Markov models $[14,16]$ and the continuous-time Markov models [5, 10] have also found applications in hierarchical manpower systems. More details on the use of manpower planning models are found in Bartholomew et al.[1]. Mathematical models for predicting the future manpower needs in the education sector were reviewed by Johnes [7]. However, the models in [7] do not take into consideration the grade-specific supervision completion rates of temporary staff (Graduate Assistants in this study), who choose to study in the institution in which they are employed for higher qualification(s) with a view to enhancing their career prospects. It is against this background we attempt to revisit manpower forecasting by taking into consideration the grade-specific supervision completion rates. It is important to mention here that predicting the future distribution of staff in a university system is an uphill task as the flows of staff in the system cannot entirely be controlled. One of such flows is wastage which may be due to resignation, death, ill-health, etc. Hence, most hierarchical models in the literature have adopted the use of mathematical expectation [1].

This study focuses on a university manpower system with a countable state space $S, 0 \notin S$. The two-way flow between the system and the outside world, due to recruitment and wastage, is introduced by constructing a sample space $\Omega=0 \cup S$ with 0 being a state external to the system. The total number of transitions in $\Omega$ is $(\#(\Omega))^{2}$, where \# is used to denote the cardinality of a set. Nonetheless, we place a constraint on the transition process by assuming a scenario where the transitions follow a natural order, that is, from one grade to either itself or the next higher grade without demotion or double promotion. This constrain is representative of the kind of data in a Nigerian university setting. We view the manpower system of the university as a random process made up of stocks and flows in a three-dimensional space $\mathbb{R}^{3}$ according to $\#(S)=3$. This is in line with the staff-mix by rank of the National Universities Commission [3, 13]. Considering a grade-specific supervision completion rates for the Graduate Assistants to obtain a higher degree (usually, a master's degree) from the university of employment within a specific time bound and that recruitment is done to replace leavers and to achieve the desired growth $[4,15]$, we develop a recurrence system where a linear mapping $F: \mathbb{R}^{3} \rightarrow \mathbb{R}^{3}$ is capable of predicting the future academic staff structure. The basic properties of the transformation matrix of the linear mapping are given with proofs. The transformation matrix has a stable set of parameters.

\subsection{A brief description}

It is rather useful to write something about the university system, which suits the Nigerian university setting considered in this paper. Academic staff in the university system is stratified into three states: the professorial cadre, which is made up of Professors and Associate Professors; the cadre of senior lecturers; and the junior academic staff cadre comprising the rank of Lecturer I and below, excluding the position of Graduate Assistant. This kind of stratification is being adopted by the National 
Universities Commission [13]. Thus academic staff may be listed from the rank of Assistant Lecturer to the rank of Professor. The rank may be used as a determinant of a staff commitment [11]. The position of Graduate Assistant in Nigerian universities is a temporary appointment for academic staff without a master's degree. The terms and conditions for this appointment have a clause which stipulates a maximum time bound (usually four calendar years) for the staff to obtain a higher degree, otherwise such a staff losses the position. A Graduate Assistant (GA) may either enrol for a higher degree programme in the university of employment or apply for a leave of absence to study elsewhere. In either case, the maximum time bound must not be exceeded. In the first case, the tuition fee is borne by the university and the staff are under the tutelage of the members of the Postgraduate Board of Studies and Examiners. In most university settings, the task of postgraduate (PG) supervision is limited to staff in the rank of Senior Lecturer and the professorial cadre. Thus, a member of the PG board contributes a certain number of assistant lecturers to the junior academic staff cadre. The size of the PG board has great implications on the number of supervisors and candidates admitted into higher degree programmes [12]. Each member of academic staff holds a membership, which is given up when the staff leaves the university (due to resignation, retirement, retrenchment, etc.) and is taken by new recruits into the system. The progression of academic staff through the ranks in the university is normally by promotion and upgrading. Upgrading applies to staff who possesses higher qualification(s) to move to the next higher rank without necessarily completing the length of service interval for promotion, while promotion is a move to the next higher rank after satisfying certain requirements and completing the length of service interval.

\subsection{Basic definitions}

To make this study clearer to a broader audience, we define some key terms and notations. The definitions are credited to [2]. Except where otherwise stated, this study assumes that $\mathbf{A}$ is an $n \times n$ square matrix.

Definition 1.2.1. The set $\operatorname{sp}(\mathbf{A})=\{\lambda \in \mathbf{C}: \operatorname{det}(\mathbf{A}-\lambda \mathbf{I})=0\}$ forms the set of eigenvalues of matrix $\mathbf{A}$ and $\operatorname{sp}(\mathbf{A})$ is called the spectrum of matrix $\mathbf{A}$.

Definition 1.2.2. The real number $\rho(\mathbf{A})=\max \{|\lambda|: \lambda \in \operatorname{sp}(\mathbf{A})\}$ is called the spectral radius of matrix $\mathbf{A}$.

Definition 1.2.3. An eigenvalue of algebraic multiplicity unity is called simple; otherwise it is said to be multiple.

Definition 1.2.4. An eigenvalue of multiplicity $m$ greater than unity is said to be semi-simple if it admits $m$ linearly independent eigenvectors $\mathbf{0} \neq \mathbf{X} \in \mathbf{C}^{n}$ such that $\mathbf{A X}=\lambda \mathbf{X}$; otherwise it is said to be defective.

Definition 1.2.5. A diagonalisable matrix is a matrix whose eigenvalues are semisimple.

Definition 1.2.6. A matrix is said to be non-negative if the elements are either positive or zero. 


\section{Methodology}

This section contains theorems on the recurrence system for the academic staff structure, the basic properties of the transformation matrix and some remarks.

\subsection{Model development}

We consider a university system with a stable manpower expansion rate. Let the evolution of academic staff structure for the university be required at the end of a period of length $\Delta$ years from an interval of the form $[0, \Delta),[\Delta, 2 \Delta), \cdots,[(\beta-1) \Delta, \beta \Delta)$, $\beta \geq 1$. More specifically, we choose $\Delta$ to coincide with the maximum length of stay for GA's. In most universities in Nigeria such as the University of Benin, $\Delta=4$. Let $t$ be a measure of time in a discrete time scale of a multiple of $\Delta$ years. Let $S=\{1,2,3\}$ be a state space for the university system, where state 1 stands for the junior academic staff cadre, state 2 for the cadre for senior lecturers and state 3 for the professorial cadre. The states are assumed to be mutually exclusive and exhaustive. Let $\mathbf{n}(t) \in \mathbb{R}^{3}$ be a column vector with entries denoted as $n_{i}(t)$ being the number of staff in state $i \in S$ at time $t$. The number $n_{i}(t)$ is the manpower stock in state $i$. The vector $\mathbf{n}(t)$ is called the academic staff structure (or grade structure) at time $t$. The initial academic staff structure is assumed known (that is, no 'ghost' worker) and it is denoted as $\mathbf{n}(0)$. The realisation of the system at time $t$ is the total number of staff given as $N(t)=\mathbf{e n}(t)$, where $\mathbf{e} \in \mathbb{R}^{3}$ is a row vector of ones. The flow of staff from a state $i$ to a state $j=i, i+1 \in S$, in a given period $t$ is assumed to be a random variable with a binomial distribution and the parameters of the distribution are the stock in state $i$ and the transition probability from state $i$ to state $j$ in period $t$.

Let $p_{i j}(t)$ be the transition probability from state $i$ to $j$ in period $t, i, j \in S$. We assume a system with a stable set of parameters and policies so that $p_{i j}(t)=p_{i j}$ for all $t$. This assumption was earlier employed by Nilakantan [11]. The transition probabilities satisfy the relation $p_{i 1}+p_{i 2}+p_{i 3} \leq 1$ for each $i \in S$, owing to the loss of staff. Let $m_{j}$ denote a grade-specific supervision completion rate for GA's assigned to a supervisor in state $j>1$ in the interval $[t, t+\Delta]$. The $m_{j}$ 's are assumed constant in time. Since PG supervision is restricted to members of the PG board, then $m_{1}=0$ and $m_{2}, m_{3} \in \mathbb{R}$. The number of GA's upgraded to the rank of Assistant Lecturer in an interval of length $\Delta$, which in turn expands the junior academic staff cadre, is given by $m_{j} n_{j}(t)$. We treat GA's who have obtained a higher degree outside their university of employment as a part of new recruits into the junior academic staff cadre. Let $p_{0 j}$ denote the fraction of recruits into category $j \in S$, where $0 \notin S$ is a state where staff who leave the system are transferred. Let $g$ denote the desired expansion rate for the system. We assume that recruitment to reach the desired expansion rate is done independently of internal movements. By the assumption that recruitment is done to replace wastage and to achieve the desired expansion [15], the number of recruits into a state $i$ in the next period is obtained as

$$
\sum_{i=1}^{3}\left(1-p_{i i}-p_{i j}\right) n_{i}(t)+g\left(\sum_{i=1}^{3} n_{i}(t)\right), i, j=i+1 \in S, \quad p_{34}=0 .
$$


With the aforementioned assumptions and notations at hand, we give the main theorem to provide a tool for practical applications.

Theorem 2.1.1. Let there be a university system, where the academic staff are stratified into three states and temporary recruits as Graduate Assistants may choose to study in the university in which they are employed. Assuming a stable set of parameters and policies and that recruitment is done to replace wastage and to achieve the desired expansion, the evolution of staff structures in the university is a linear recurrence system of the form

$$
\boldsymbol{n}(t+\Delta)=\boldsymbol{A} \boldsymbol{n}(t), \quad t=0, \Delta, 2 \Delta, \cdots
$$

up to the latest time we wish to project to, with $\boldsymbol{A}=\boldsymbol{Q}^{\prime} \in \mathbb{R}^{3 \times 3}$, where the entries $q_{i j}$ in $\boldsymbol{Q}$ are defined by

$$
q_{i j}=\left\{\begin{array}{cc}
\phi_{i j}+p_{0 j}\left(1-p_{i i}-p_{i, i+1}+g\right) & \text { for } i \neq 3 \\
\phi_{i j}+p_{0 j}\left(1-p_{i i}+g\right) & \text { for } i=3,
\end{array}\right.
$$

$\phi_{i j}=p_{i j}$ for $j=i, i+1, \phi_{i 1}=m_{i}$ for $i>1, \phi_{13}=\phi_{32}=0$, and the prime' denotes a matrix transpose.

Proof. Consider the accounting equation for each state $i$ in $S$ of the system:

$$
\begin{gathered}
n_{1}(t+\Delta)=\sum_{i=1}^{3} \phi_{i 1} n_{i}(t)+p_{01}\left(\sum_{i=1}^{3}\left(1-p_{i i}-p_{i j}\right) n_{i}(t)+g\left(\sum_{i=1}^{3} n_{i}(t)\right)\right), \\
n_{2}(t+\Delta)=p_{12} n_{1}(t)+p_{22} n_{2}(t)+p_{02}\left(\sum_{i=1}^{3}\left(1-p_{i i}-p_{i j}\right) n_{i}(t)+g\left(\sum_{i=1}^{3} n_{i}(t)\right)\right),
\end{gathered}
$$

and

$$
n_{3}(t+\Delta)=p_{23} n_{2}(t)+p_{33} n_{3}(t)+p_{03}\left(\sum_{i=1}^{3}\left(1-p_{i i}-p_{i j}\right) n_{i}(t)+g\left(\sum_{i=1}^{3} n_{i}(t)\right)\right) .
$$

Rewriting the accounting equations as a single matrix equation, we get

$$
\mathbf{n}(t+\Delta)=\mathbf{L}^{\prime} \mathbf{n}(t)+\mathbf{P}_{0}^{\prime}\left(\mathbf{e}\left[\mathbf{I}-\mathbf{P}^{\prime}\right] \mathbf{n}(t)+g \mathbf{e n}(t)\right),
$$

where

$$
\mathbf{L}^{\prime}=\left[\begin{array}{ccc}
p_{11} & m_{2} & m_{3} \\
p_{12} & p_{22} & 0 \\
0 & p_{23} & p_{33}
\end{array}\right] \text { is a } 3 \times 3 \text { Leslie matrix, } \mathbf{P}_{0}^{\prime}=\left[\begin{array}{c}
p_{01} \\
p_{02} \\
p_{03}
\end{array}\right] \text { is a } 3 \times 1
$$

recruitment vector, $\mathbf{P}^{\prime}=\left[\begin{array}{ccc}p_{11} & 0 & 0 \\ p_{12} & p_{22} & 0 \\ 0 & p_{23} & p_{33}\end{array}\right]$ is a $3 \times 3$ sub-stochastic transition 
matrix, $\mathbf{e}=\left[\begin{array}{lll}1 & 1 & 1\end{array}\right]$ and

$$
\mathbf{I}=\left[\delta_{i j}\right] \text { with } \delta_{i j}=\left\{\begin{array}{cc}
1 & \text { for } i=j \\
0 & \text { for } i \neq j,
\end{array} i, j \in S .\right.
$$

The vector $\mathbf{L}^{\prime} \mathbf{n}(t)+\mathbf{P}_{0}^{\prime}\left(\mathbf{e}\left[\mathbf{I}-\mathbf{P}^{\prime}\right] \mathbf{n}(t)+g \mathbf{e n}(t)\right)$ simplifies to $\left(\mathbf{L}^{\prime}+\mathbf{P}_{0}^{\prime} \mathbf{e}[(1+g) \mathbf{I}\right.$ $\left.\left.-\mathbf{P}^{\prime}\right]\right) \mathbf{n}(t)$, where the matrix $\left(\mathbf{L}^{\prime}+\mathbf{P}_{0}^{\prime} \mathbf{e}\left[(1+g) \mathbf{I}-\mathbf{P}^{\prime}\right]\right)=\mathbf{Q}^{\prime}$. This completes the proof.

\subsection{Basic properties}

Theorem 2.1.1 above provides a recurrence framework to describe the academic staff structure of a university system. The behaviour of the system over time depends on the nature of matrix A. Clearly, matrix $\mathbf{A}$ is a non-negative matrix. Nonetheless, there are other interesting properties of matrix $\mathbf{A}$. We state these properties as propositions with proofs.

Proposition 2.2.1. The eigenvalue $\lambda \in s p(\mathbf{A}) \subset \mathbf{C}$ is given by

$$
\lambda=\left\{\begin{array}{cl}
\frac{1}{3} \operatorname{tr}(\mathbf{A})+\left(\operatorname{det}(\mathbf{A})-\frac{1}{27} \operatorname{tr}^{3}(\mathbf{A})\right)^{\frac{1}{3}} \exp \left(z\left(\frac{2}{3}(k+1) \pi\right)\right) & \text { if } \operatorname{tr}^{2}(\mathbf{A})=3 S_{2} \\
\frac{1}{3} \operatorname{tr}(\mathbf{A})+r \cos \left(\frac{2 k \pi}{3}+\frac{1}{3} \arccos \zeta\right) & \text { if } \operatorname{tr}^{2}(\mathbf{A}) \neq 3 S_{2},
\end{array}\right.
$$

where $k=0,1,2, z=\sqrt{-1}, \operatorname{tr}(\mathbf{A})$ is the trace of matrix $\mathbf{A}, S_{2}$ is the sum of the principal minors of matrix $\mathbf{A}$ of order $2, r=\frac{2}{3}\left(t^{2}(\mathbf{A})-3 S_{2}\right)^{\frac{1}{2}}$, and

$$
\zeta=\frac{27\left(\operatorname{det}(\mathbf{A})-\left(\frac{1}{3} \operatorname{tr}(\mathbf{A})\right)^{3}\right)+3 \operatorname{tr}(\mathbf{A})\left(\operatorname{tr}^{2}(\mathbf{A})-3 S_{2}\right)}{2\left(\operatorname{tr}^{2}(\mathbf{A})-3 S_{2}\right)^{\frac{3}{2}}} .
$$

Proof. Let $P(\lambda)$ denote the characteristic polynomial of matrix $\mathbf{A}$, which is expressed as [9]

$$
P(\lambda)=\lambda^{3}-\operatorname{tr}(\mathbf{A}) \lambda^{2}+S_{2} \lambda-\operatorname{det}(\mathbf{A}) .
$$

Since the eigenvalues $\lambda \in s p(\mathbf{A})$ are obtained by solving the singular pencil $\operatorname{det}(\mathbf{A}-$ $\lambda \mathbf{I})=0$, we set

$$
\lambda^{3}-\operatorname{tr}(\mathbf{A}) \lambda^{2}+S_{2} \lambda-\operatorname{det}(\mathbf{A})=0 .
$$

Let $\lambda=x+\frac{1}{3} \operatorname{tr}(\mathbf{A})$. Then

$$
x^{3}+\left(S_{2}-\frac{1}{3} \operatorname{tr}^{2}(\mathbf{A})\right) x+\frac{1}{3} \operatorname{tr}(\mathbf{A})\left(S_{2}-\frac{2}{9} \operatorname{tr}^{2}(\mathbf{A})\right)-\operatorname{det}(\mathbf{A})=0 .
$$

If $\operatorname{tr}^{2}(\mathbf{A})=3 S_{2}$, then

$$
x=\left(\operatorname{det}(\mathbf{A})-\frac{1}{27} \operatorname{tr}^{3}(\mathbf{A})\right)^{\frac{1}{3}},\left(\operatorname{det}(\mathbf{A})-\frac{1}{27} \operatorname{tr}^{3}(\mathbf{A})\right)^{\frac{1}{3}}\left(\cos \frac{2 \pi}{3} \pm z \sin \frac{2 \pi}{3}\right) .
$$


Using the exponential form of a complex number, we obtain

$$
\lambda=\frac{1}{3} \operatorname{tr}(\mathbf{A})+\left(\operatorname{det}(\mathbf{A})-\frac{1}{27} \operatorname{tr}^{3}(\mathbf{A})\right)^{\frac{1}{3}} \exp \left(z\left(\frac{2}{3}(k+1) \pi\right)\right), \quad k=0,1,2 .
$$

On the other hand, if $\operatorname{tr}^{2}(\mathbf{A}) \neq 3 S_{2}$, then we set $x=r \cos \theta$ and then compare the resulting equation

$$
r^{3} \cos ^{3} \theta+\left(S_{2}-\frac{1}{3} t^{2}(\mathbf{A})\right) r \cos \theta=\operatorname{det}(\mathbf{A})-\frac{1}{3} \operatorname{tr}(\mathbf{A})\left(S_{2}-\frac{2}{9} \operatorname{tr}^{2}(\mathbf{A})\right)
$$

with the trigonometric identity

$$
4 \cos ^{3} \theta-3 \cos \theta=\cos 3 \theta
$$

Thus,

Further simplifications yield

$$
\frac{r^{3}}{4}=\frac{\left(\frac{1}{3} t r^{2}(\mathbf{A})-S_{2}\right) r}{3}=\frac{\operatorname{det}(\mathbf{A})-\frac{1}{3} \operatorname{tr}(\mathbf{A})\left(S_{2}-\frac{2}{9} t r^{2}(\mathbf{A})\right)}{\cos 3 \theta} .
$$

$$
\begin{gathered}
r=0, \quad \pm \frac{2}{3}\left(t r^{2}(\mathbf{A})-3 S_{2}\right)^{\frac{1}{2}}, \text { and } \\
\cos 3 \theta=\frac{3\left(\operatorname{det}(\mathbf{A})-\left(\frac{1}{27} \operatorname{tr}(\mathbf{A})\right)^{3}\right)+\frac{1}{3} \operatorname{tr}(\mathbf{A})\left(t^{2}(\mathbf{A})-3 S_{2}\right)}{\left(\frac{1}{3} t r^{2}(\mathbf{A})-S_{2}\right) r} .
\end{gathered}
$$

We exclude the case $r=0$ as division by zero is undefined. With

$$
r=\frac{2}{3}\left(t^{2}(\mathbf{A})-3 S_{2}\right)^{\frac{1}{2}}
$$

we find

$$
\theta=\frac{2 k \pi}{3}+\frac{1}{3} \arccos \left(\frac{27\left(\operatorname{det}(\mathbf{A})-\left(\frac{1}{3} \operatorname{tr}(\mathbf{A})\right)^{3}\right)+3 \operatorname{tr}(\mathbf{A})\left(\operatorname{tr}^{2}(\mathbf{A})-3 S_{2}\right)}{2\left(\operatorname{tr}^{2}(\mathbf{A})-3 S_{2}\right)^{\frac{3}{2}}}\right)
$$

$k=0,1,2$. The case $r=-\frac{2}{3}\left(t^{2}(\mathbf{A})-3 S_{2}\right)^{\frac{1}{2}}$ reproduces the same eigenvalues as with $r=\frac{2}{3}\left(t r^{2}(\mathbf{A})-3 S_{2}\right)^{\frac{1}{2}}$.

Proposition 2.2.2. For a non-contracting system, the trace $\operatorname{tr}(\mathbf{A})$ lies in the interval $[0,9)$.

Proof. By definition of the trace of a matrix,

$$
\begin{aligned}
\operatorname{tr}(\mathbf{A})=p_{11}+p_{01}\left(1-p_{11}-p_{12}+g\right)+p_{22}+p_{02}(1 & \left.-p_{22}-p_{23}+g\right) \\
& +p_{33}+p_{03}\left(1-p_{33}+g\right) .
\end{aligned}
$$


Since $p_{i j}, i, j \in S$, is a transition probability, $0 \leq p_{i j} \leq 1$. Furthermore, $\left(1-p_{i i}-p_{i, i+1}\right)$ and $p_{0 j}$ are respectively wastage and recruitment probabilities; so they lie in the interval $[0,1]$. For $0 \leq g<1, \operatorname{tr}(\mathbf{A}) \geq 0$ and $p_{i i}+p_{0 j}\left(1-p_{i i}-p_{i, i+1}+g\right)<3$ for each $i=j \in S$. Hence $0 \leq \operatorname{tr}(\mathbf{A})<9$.

Proposition 2.2.3. The spectral radius $\rho(\mathbf{A})$ satisfies the relation $\rho(\mathbf{A})<\Psi$, where

$$
\Psi= \begin{cases}3+\left|\operatorname{det}(\mathbf{A})-\frac{1}{27} \operatorname{tr}^{3}(\mathbf{A})\right|^{\frac{1}{3}} & \text { if } \operatorname{tr}^{2}(\mathbf{A})=3 S_{2} \\ 3+|r|\left(\cosh ^{2} b-\sin ^{2} a\right)^{\frac{1}{2}} & \text { if } \operatorname{tr}^{2}(\mathbf{A}) \neq 3 S_{2},\end{cases}
$$

and the argument $\left(\frac{2 k \pi}{3}+\frac{1}{3} \arccos \zeta\right)$ in Proposition 2.2.1 is of the form $a+z b \in \mathbf{C}$, $a, b \in \mathbf{R}$.

Proof. The proof follows from Propositions 2.2.1 and 2.2.2. If $\operatorname{tr}^{2}(\mathbf{A})=3 S_{2}$, then

$$
\begin{aligned}
|\lambda| \leq\left|\frac{1}{3} \operatorname{tr}(\mathbf{A})\right|+ & \left|\left(\operatorname{det}(\mathbf{A})-\frac{1}{27} \operatorname{tr}^{3}(\mathbf{A})\right)^{\frac{1}{3}} \exp \left(z\left(\frac{2}{3}(k+1) \pi\right)\right)\right| \\
& <3+\left|\operatorname{det}(\mathbf{A})-\frac{1}{27} \operatorname{tr}^{3}(\mathbf{A})\right|^{\frac{1}{3}} .
\end{aligned}
$$

On the other hand, if $\operatorname{tr}^{2}(\mathbf{A}) \neq 3 S_{2}$, then

$$
|\lambda| \leq\left|\frac{1}{3} \operatorname{tr}(\mathbf{A})\right|+\left|r \cos \left(\frac{2 k \pi}{3}+\frac{1}{3} \arccos \zeta\right)\right|
$$

It follows that

$$
|\lambda|<3+|r||\cos a \cosh b-z \sin a \sinh b| \text { as }\left(\frac{2 k \pi}{3}+\frac{1}{3} \arccos \zeta\right)=a+z b .
$$

Thus

$$
|\lambda|<3+|r|\left(\cos ^{2} a \cosh ^{2} b+\sin ^{2} a \sinh ^{2} b\right)^{\frac{1}{2}}=3+|r|\left(\cosh ^{2} b-\sin ^{2} a\right)^{\frac{1}{2}} .
$$

Proposition 2.2.4. If $\operatorname{det}(\mathbf{A}) \neq \frac{1}{27} t r^{3}(\mathbf{A})$, then the transformation matrix $\mathbf{A}$ is diagonalisable over the complex field $\mathbf{C}$.

Proof. If $\operatorname{det}(\mathbf{A}) \neq \frac{1}{27} \operatorname{tr}^{3}(\mathbf{A})$, then, from Proposition 2.2.1, $\lambda$ is simple as its algebraic multiplicity is unity. Since $\lambda \in \mathbf{C}$, matrix $\mathbf{A}$ is diagonalisable over the complex field $\mathbf{C}$. 


\subsection{Some remarks}

We make some remarks on the staff structure $\mathbf{n}(t+\Delta)$ and its representation.

Remark 2.3.1. On examining the structure $\boldsymbol{n}(t+\Delta)$ as $t \rightarrow \infty$, Proposition 2.2.3 indicates that the system may be unstable and $\boldsymbol{n}(t+\Delta)$ may be unbounded.

Remark 2.3.2. Proposition 2.2.4 implies that the linear recurrence system given in Theorem 2.1.1 may be written as

$$
\boldsymbol{n}(0), \quad \boldsymbol{n}(\beta \Delta)=\boldsymbol{X} \boldsymbol{D}^{\beta} \boldsymbol{X}^{-1} \boldsymbol{n}(0), \quad \beta=1,2,3, \cdots,
$$

according to $t=0, \Delta, 2 \Delta, \cdots$, where $\boldsymbol{D}=\operatorname{diag}\left(\lambda_{1}, \lambda_{2}, \lambda_{3}\right), \lambda_{i} \in \operatorname{sp}(\boldsymbol{A}) \subset \boldsymbol{C}, i=$ $1,2,3$, and the ith column of $\boldsymbol{X}$ is the right eigenvector $\boldsymbol{X}_{i}$ associated with the eigenvalue $\lambda_{i}$.

Remark 2.3.2 enables us to make the following claim.

Theorem 2.3.1. Let the transformation matrix, $\boldsymbol{A}$, be diagonalisable over the complex field, $\boldsymbol{C}^{3 \times 3}$. Then the manpower structure can be determined at any instant of time in the complex field.

Proof. For $\lambda_{i} \in \operatorname{sp}(\mathbf{A}) \subset \mathbf{C}, i \in S, \lambda_{i}^{\frac{\beta}{\alpha}}$ is defined in $\mathbf{C}$, for all $\alpha, \beta \in \mathbb{R}$. Hence $\mathbf{X}$, $\operatorname{diag}\left(\lambda_{1}^{\frac{\beta}{\alpha}}, \lambda_{2}^{\frac{\beta}{\alpha}}, \lambda_{3}^{\frac{\beta}{\alpha}}\right), \mathbf{X}^{-1} \in \mathbf{C}^{3 \times 3}$ and $\mathbf{n}\left(\frac{\beta}{\alpha} \Delta\right) \in \mathbf{C}^{3}$.

Remark 2.3.3. In practice, the structure $\boldsymbol{n}\left(\frac{\beta}{\alpha} \Delta\right)$ is required in $\mathbb{R}^{3}$ and as a nonnegative integer. For this reason, the modulus of the entries in $\boldsymbol{n}\left(\frac{\beta}{\alpha} \Delta\right)$ to the nearest integer should be used in real-life applications.

\section{Numerical Illustration}

We illustrate the previous results in the Methodology with an example from a department in a university in Nigeria. We carryout all computations in the MATLAB environment. From the records [3], the sub-stochastic transition matrix is

$$
\mathbf{P}=\left[\begin{array}{ccc}
\frac{70}{83} & \frac{12}{83} & 0 \\
0 & \frac{37}{46} & \frac{9}{46} \\
0 & 0 & \frac{33}{34}
\end{array}\right]
$$

The time interval for GA's to obtain a higher degree from the university is 4 years. For this reason, we set the length of the interval, $\Delta$, as 4 years. Let the gradespecific supervision completion rates for GA's within the time bound be $m_{2}=0.4$ 
and $m_{3}=1.2$. Suppose the system maintains a growth rate of $1 \%$ and adopts a recruitment policy of $p_{01}=0.6, p_{02}=0.4$ and $p_{03}=0$. Then the transformation matrix $\mathbf{A}$ is

$$
\mathbf{A}=\left[\begin{array}{ccc}
0.8566 & 0.4060 & 1.2236 \\
0.1534 & 0.8083 & 0.0158 \\
0 & 0.1957 & 0.9706
\end{array}\right]
$$

In order to evaluate $s p(\mathbf{A})$, we first compute the coefficients of the characteristic equation as $\operatorname{tr}(\mathbf{A})=2.6355, S_{2}=2.2430$ and $\operatorname{det}(\mathbf{A})=0.6457$. The trace $\operatorname{tr}(\mathbf{A})$ lies within the interval defined by Proposition 2.2.2. Since $\operatorname{tr}^{2}(\mathbf{A})>3 S_{2}$, we determine $\lambda$ from the formula

$$
\lambda=\frac{1}{3} \operatorname{tr}(\mathbf{A})+r \cos \left(\frac{2 k \pi}{3}+\frac{1}{3} \arccos \zeta\right)
$$

in Proposition 2.2.1. Thus we obtain

$$
s p(\mathbf{A})=\{1.2687,0.6834-0.2047 z, 0.6834+0.2047 z\} .
$$

Since the eigenvalues of $\mathbf{A}$ are simple, matrix $\mathbf{A}$ is diagonalisable over the complex field $\mathbf{C}$. This is in line with Proposition 2.2.4. The matrix A may be expressed as

$$
\mathbf{A}=\mathbf{X D X}^{-1}
$$

where

$$
\begin{aligned}
& \mathbf{X}=\left[\begin{array}{ccc}
0.9260 & -0.8080 & -0.8080 \\
0.3156 & 0.2523+0.4492 z & 0.2523-0.4492 z \\
0.2071 & 0.0306-0.2842 z & 0.0306+0.2842 z
\end{array}\right], \\
& \mathbf{D}=\left[\begin{array}{ccc}
1.2687 & 0 & 0 \\
0 & 0.6834+0.2047 z & 0 \\
0 & 0 & 0.6834-0.2047 z
\end{array}\right],
\end{aligned}
$$

and

$$
\mathbf{X}^{-1}=\left[\begin{array}{ccc}
0.3768 & 1.0124 & 1.6003 \\
-0.4029-0.0939 z & 0.5801-0.4315 z & 0.9170+1.0773 z \\
-0.4029+0.0939 z & 0.5801+0.4315 z & 0.9170-1.0773 z
\end{array}\right]
$$

The spectral radius of matrix $\mathbf{A}$, which is $\rho(\mathbf{A})=1.2687$, does not exceed the upper bound $(3+|0.3105 \cos (0+0.7021 z)|)=3.3902$ as specified in Proposition 2.2.3. The value of $\rho(\mathbf{A})$ shows that $\lim _{\beta \rightarrow \infty} \mathbf{A}^{\beta}$ is infinitely large. Thus the grade structure $\mathbf{n}(t+\Delta)$ is unbounded as $t$ becomes very large. Now, let the initial grade structure for the system be $\mathbf{n}(0)=[14,6,12]^{\prime}$. The grade structure at $t=0, \Delta$, are $\mathbf{n}(\Delta)=$ $[29,7,13]^{\prime}$ and $\mathbf{n}(2 \Delta)=[44,10,14]^{\prime}$. Now, suppose the structure of the system is required at a period $4 \Delta / 3$. Then $\mathbf{n}(4 \Delta / 3)=[34,8,13]^{\prime}$. The realisation of the process satisfies the relation $N(\Delta)<N(4 \Delta / 3)<N(2 \Delta)$ as the system is an expanding one. 


\section{Conclusion}

This study provides a means of predicting a university manpower structure. The study provided a new representation for the transformation matrix of the system. A considerable amount of effort has been devoted to the properties of the transformation matrix. The transformation matrix is equivalent to the transition matrix in the manpower literature when the grade-specific supervision completion rates are equal to zero, that is $m_{2}=m_{3}=0$. The introduction of the grade-specific supervision completion rates, $m_{2}, m_{3}$, gives a better insight into the phenomenon and the output of supervisors on GA's. We opined that our model, which is a linear recurrence system, does succeed in unifying the stocks and flows of academic staff in a university system. The study is easy to follow and the theoretical sophistication is minimal. However, further work may be required to study the statistical properties of the linear recurrence system.

\section{References}

[1] D.J. Bartholomew, A.F. Forbes, S.I. McClean, Statistical Techniques for Manpower Planning (2nd Ed.), Chichester: John Wiley \& Sons, 1991.

[2] F. Chatelin, Eigenvalues of Matrices: Revised Edition, Classics in Applied Mathematics, SIAM, 2012.

[3] V.U. Ekhosuehi, A.A. Osagiede, W.A. Iguodala, A procedure for distributing recruits in manpower systems, Accepted for publication in Yugoslav Journal of Operations Research 24 (2) (2015). DOI: 10.2298/YJOR131219031E.

[4] V.U. Ekhosuehi, A.A. Osagiede, Evolution of Structures in a University System, Scholar's Press, Saarbrucken, 2015.

[5] I. Gerontidis, On certain aspects of non-homogeneous Markov systems in continuous time, Journal of Applied Probability 27 (3) (1990) 530-544.

[6] M.A. Guerry, Properties of calculated predictions of graded sizes and the associated integer valued vectors, Journal of Applied Probability 34 (1) (1997) 94-100.

[7] J. Johnes, Operational research in education, European Journal of Operational Research (2014). doi: 10.1016/j.ejor.2014.10.043.

[8] I. Kipouridis, G. Tsaklidis, The size order of the state vector of discrete-time homogeneous Markov systems, Journal of Applied Probability 38 (2) (2001) 357368 .

[9] S. Lipschutz, M. Lipson, Schaum's Outlines: Linear Algebra (Fourth Edition), McGraw-Hill, 2009. 
[10] S. McClean, E. Montgomery, F. Ugwuowo, Non-homogeneous continuous-time Markov and semi-Markov manpower models, Applied Stochastic Models and Data Analysis 13 (1998) 191-198.

[11] K. Nilakantan, Evaluation of staffing policies in Markov manpower systems and their extension to organizations with outsource personnel, Journal of the Operational Research Society 66 (2015) 1324-1340.

[12] M.G. Nicholls, The use of Markov models as an aid to the evaluation, planning and benchmarking of doctoral programs, Journal of the Operational Research Society 60 (2009) 1183-1190.

[13] O. Osasona, Tools for academic planning, In: Uvah, I. I. (Editor), Practical Guide on Academic Planning in Nigerian Universities: A Compendium of Academic Planning Tools (2012) 62-105.

[14] F.I. Ugwuowo, S.I. McClean, Modelling heterogeneity in a manpower system: a review, Applied Stochastic Models in Business and Industry 16 (2000) 99-110.

[15] P.-C.G. Vassiliou, On the periodicity of non-homogeneous Markov chains and systems, Linear Algebra and its Applications 471 (2015) 654-684.

[16] P.-C.G. Vassiliou, A.A. Papadopoulo, Non-homogeneous semi-Markov systems and maintainability of the state sizes, Journal of Applied Probability 29 (1992) 519-534.

[17] P.-C.G. Vassiliou, N. Tsantas, Maintainability of structures in nonhomogeneous Markov systems under cyclic behaviour and input control, SIAM Journal on Applied Mathematics 44 (5) (1984) 1014-1022.

\section{DOI: $10.7862 / \mathrm{rf} .2016 .3$}

\section{Virtue U. Ekhosuehi}

email: virtue.ekhosuehi@uniben.edu

Department of Mathematics, University of Benin,

Benin City, Edo State, Nigeria.

Received 24.12.2015

Accepted 18.03.2016 